\title{
DWT Based-Approach for Color Image Compression Using Genetic Algorithm
}

\author{
Aldjia Boucetta $^{1}$ and Kamal Eddine Melkemi ${ }^{2}$ \\ 1 Department of Computer Science, Faculty of Science, University of Batna, \\ 05000 Batna, Algeria \\ boucetta_batna@yahoo.fr \\ 2 Department of Computer Science, Faculty of Science, University of Biskra, \\ 07000 Biskra, Algeria \\ melkemi2002@yahoo.com
}

\begin{abstract}
This paper describes a color image compression technique based on Discrete Wavelet Transform (DWT) and Genetic Algorithm (GA). High degree of correlation between the RGB planes of a color image is reduced by transforming them to more suitable space by using the GA. This GA would enable us to find $\mathrm{T}_{1} \mathrm{~T}_{2} \mathrm{~T}_{3}$ representation, in which $T_{1}$ energy is more maximized than that of $T_{2}$ and $T_{3}$.

The result of the proposed method is compared with previous similar published methods and the former is found superior in terms of quality of the reconstructed image.

Further, proposed method is efficient in compression ability and fast in implementation.
\end{abstract}

Keywords: Color image compression, Color space, Discrete wavelet transform, Arithmetic encoder, Two-role encoder, Genetic algorithm.

\section{Introduction}

Compression/coding of digital image is done by detecting and removing redundant information from the image. Image compression algorithm consists of two basic categories:

Methods of the first category are called direct image compression [1, [2] methods which are applied directly on the samples of an image in the spatial domain. Block Truncation Coding (BTC) and vector quantization are two widely used spatial domain compression techniques [3].

The second category contains methods called transform methods [1, 7], which transform the image to frequency representations suitable for detecting and removing redundancies, such as Discrete Fourier Transform (DFT) 1, Discrete Cosine Transform (DCT) 22, 8] and Discrete Wavelet transform (DWT) 12].

Of all the transform methods, the wavelet transform achieves better energy compaction than the DCT and hence can help in providing better compression for the same Peak Signal to Noise Ratio (PSNR). 
A comparative study of DCT and wavelet based image coding can be found in [2].

In this paper, we propose a new color image compression method based on DWT and an appropriate GA [13, 14. The RGB system color representation is the most commonly used in computer graphics. In fact, there are an infinite number of possible color spaces instead of this common RGB channels. Many of these other color spaces are derived by applying linear functions of R, G, B.

Recently, Douak et al. 4 proposed a color image compression algorithm based on the DCT transform and the RGB to YCbCr transformation. However, in our proposed approach we move from the RGB space to more suitable space for each image, by using an appropriate GA. This suitable space is referred to as $T_{1} T_{2} T_{3}$.

Indeed, our GA would enable us to find these $\mathrm{T}_{1} \mathrm{~T}_{2} \mathrm{~T}_{3}$ color space, in which $\mathrm{T}_{1}$ energy is more maximized than that of $\mathrm{T}_{2}$ and $\mathrm{T}_{3}$. This allows a more effective compression because the information is condensed in the plan $\mathrm{T}_{1}$. Thus, compress $\mathrm{T}_{2}$ and $\mathrm{T}_{3}$ more effectively .

In the remaining of this paper, the proposed method is referred to as GADWT based compression approach.

The rest of this paper is organized as follows.

Section 2 presents fundamental and methodological concepts needed in this work, and describes the performance criteria used to elaborate the GA-DWT based compression approach. Section 3 gives more details to explain the GADWT based compression approach. Section 4 presents and discusses some experimental results. Section 5 gives a general conclusion and some ideas for future research.

\section{Basic Concepts}

\subsection{Genetic Algorithm}

A GA (see [13] and [14]) is a probabilistic research algorithm that mimics the process of natural evolution. This heuristic is routinely used to generate useful solutions to search problems such as image compression [15], 16]. GAs, which generate solutions to optimization problems using methods inspired by inheritance, mutation, selection, and crossover.

In this paper, using the GA to find the $\mathrm{T}_{1} \mathrm{~T}_{2} \mathrm{~T}_{3}$ color space, bearing in mind that $\mathrm{T}_{1}$ represents the luminance; $\mathrm{T}_{2}$ and $\mathrm{T}_{3}$ represent the chrominance as:

$$
\begin{aligned}
& \mathrm{T}_{1}=a_{11} \times \mathrm{R}+a_{12} \times \mathrm{G}+a_{13} \times \mathrm{B} . \\
& \mathrm{T}_{2}=a_{21} \times \mathrm{R}+a_{22} \times \mathrm{G}+a_{23} \times \mathrm{B} . \\
& \mathrm{T}_{3}=a_{31} \times \mathrm{R}+a_{32} \times \mathrm{G}+a_{33} \times \mathrm{B} .
\end{aligned}
$$

To solve the problem, we must find $a=a_{i j}$ that maximizes the energy in $\mathrm{T}_{1}$ than that of the two other channels $\mathrm{T}_{2}$ and $\mathrm{T}_{3}$. 
GA processes:

Figure 1 shows the GA scheme used in this approach.

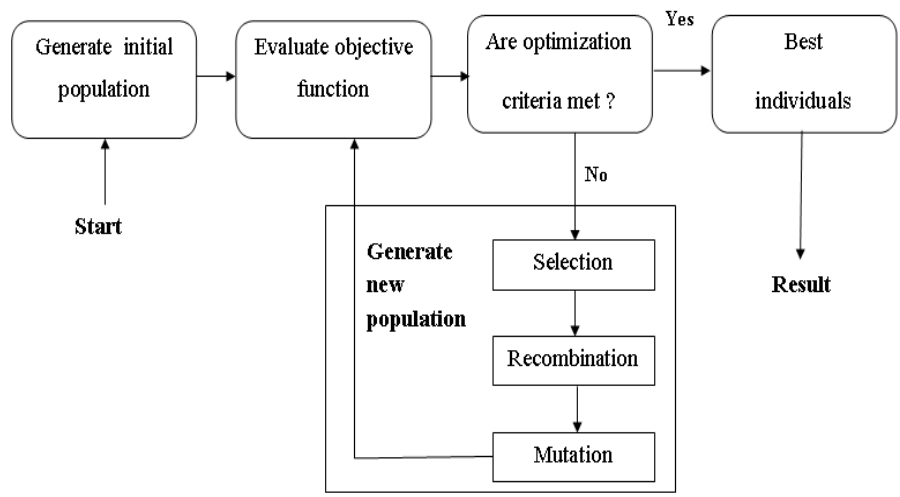

Fig. 1. The GA scheme

1. Generate Initial Population: create real-valued initial population of chromosomes. A chromosome in our algorithm is constituted by nine genes and each gene is encoded as a real number. Figure 2 shows our chromosome representation.

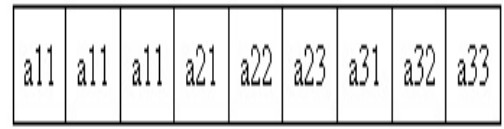

Fig. 2. The chromosome codification

2. Objective function: is used to calculate the effectiveness of each chromosome. For a more complete review, see 15.

We define $T_{1} S E, T_{2} S E$, and $T_{3} S E$, respectively, as $T_{1}, T_{2}, T_{3}$ space energy and $\mathrm{TE}_{\mathrm{T}_{1} \mathrm{~T}_{2} \mathrm{~T}_{3}}$ express the total energy. Their definitions are:

$$
\begin{gathered}
\mathrm{T}_{1} \mathrm{SE}=100 \times \frac{\sum_{i=0}^{N-1} \sum_{j=0}^{M-1} \mathrm{~T}_{1 i j}^{2}}{\mathrm{TE}_{\mathrm{T}_{1} \mathrm{~T}_{2} \mathrm{~T}_{3}}} . \\
\mathrm{T}_{2} \mathrm{SE}=100 \times \frac{\sum_{i=0}^{N-1} \sum_{j=0}^{M-1} \mathrm{~T}_{2 i j}^{2}}{\mathrm{TE}_{\mathrm{T}_{1} \mathrm{~T}_{2} \mathrm{~T}_{3}}} . \\
\mathrm{T}_{3} \mathrm{SE}=100 \times \frac{\sum_{i=0}^{N-1} \sum_{j=0}^{M-1} \mathrm{~T}_{3 i j}^{2}}{\mathrm{TE}_{\mathrm{T}_{1} \mathrm{~T}_{2} \mathrm{~T}_{3}}} . \\
\mathrm{TE}_{\mathrm{T}_{1} \mathrm{~T}_{2} \mathrm{~T}_{3}}=\sum_{i=0}^{N-1} \sum_{j=0}^{M-1} \mathrm{~T}_{1}{ }^{2}+\sum_{i=0}^{N-1} \sum_{j=0}^{M-1} \mathrm{~T}_{2}{ }^{2}+\sum_{i=0}^{N-1} \sum_{j=0}^{M-1} \mathrm{~T}_{3}{ }^{2} .
\end{gathered}
$$




$$
f=\mathrm{T}_{1} \mathrm{SE}-\left(\mathrm{T}_{2} \mathrm{SE}+\mathrm{T}_{3} \mathrm{SE}\right) .
$$

The problem is to maximize $f$.

3. Genetic Operators:

(a) Recombination (crossover): The crossover is the operator that exchanges genetic material between two individuals by selecting a point at which pieces of the parents are swapped in order to generate two new individuals [15. In our implementation we use high-level recombination operator (recombin) [19].

(b) Mutation: Mutation operator modifies the chromosome genes randomly according to the mutation probability. We use real-value mutation (mutbga) 19 .

(c) The parameters of the algorithm: The behavior of the GA can be controlled using many initial conditions and parameters. The various parameters of GA are shown in Table 1.

Table 1. Genetic algorithm parameters

\begin{tabular}{lc}
\hline Parameter & Value \\
\hline Population Size & 50 \\
Maximum generations & 20 \\
Crossover probability & 0.8 \\
Mutation probability & 0.1 \\
\hline
\end{tabular}

\subsection{Discrete Wavelet Transform}

The DWT (see [10] and [8]) is applied independently to the image components and decorrelates the image into different scale sizes, preserving much of its spatial correlation. A one-dimensional (1-D) DWT consists of a low (L) and high (H) pass filter splitting a line of pixels into two lines of half the size. Application of the filters to two-dimensional (2-D) images in horizontal and vertical directions produces four subbands (LL, LH, HL, and HH). The LL subband is a lower resolution representation of the original image, and the missing details are filtered into the remaining subbands. The subbands contain the horizontal (LH), vertical (HL), and diagonal (HH) edges on the scale size defined by the wavelet.

\subsection{Performances Criterion}

The performances of compression technique are based on two widely used essential criteria, the compression ratio, and the quality of the reconstructed image. Here, compression ratio is measured in terms of Bits Per Pixel (bpp) and the image quality in terms of PSNR [3]. The bpp is given by:

$$
\mathrm{bpp}=\frac{\text { size of compressed image in bits }}{\text { number of pixels }} .
$$


The PSNR is given by [3]:

$$
\mathrm{PSNR}=10 \times \log _{10}\left(\frac{255^{2} \times 3}{M S E(\mathrm{R})+M S E(\mathrm{G})+M S E(\mathrm{~B})}\right) .
$$

\section{GA-DWT Based Compression Approach}

The different steps of transformation, compression and decompression are summarized in Fig. 3 .

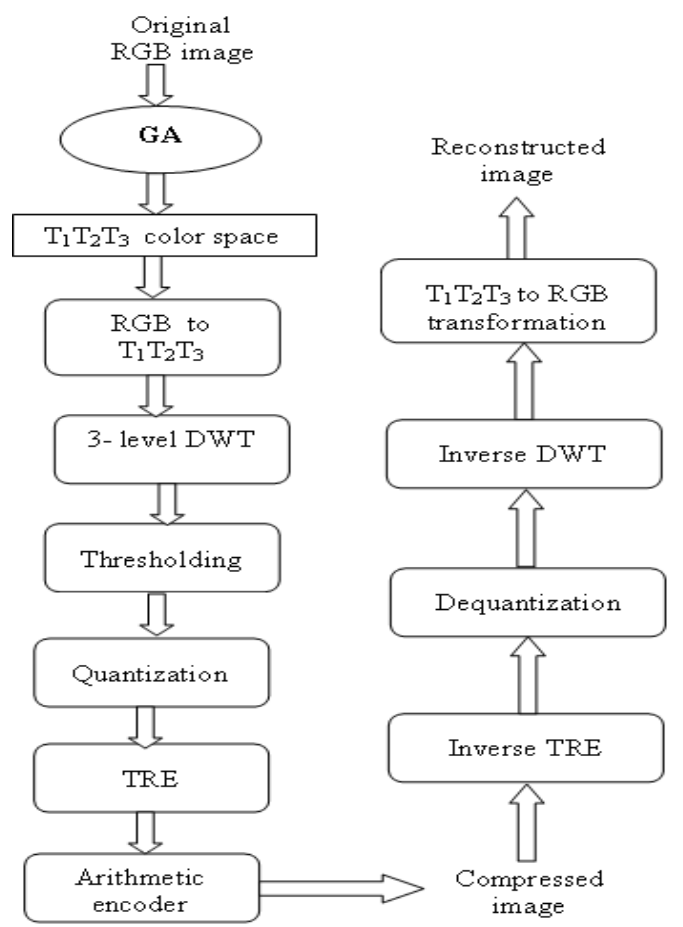

Fig. 3. The process of compression

\subsection{GA-DWT Based Compression Phase}

In this phase, the proposed GA-DWT based compression technique is built around several steps. Each step will be explained in more details as follows:

1. Genetic Algorithm: we use the GA to find the $T_{1} T_{2} T_{3}$ color space. $T_{1}$ represents luminance information and $\mathrm{T}_{2}, \mathrm{~T}_{3}$ are chrominance information.

2. RGB to T1T2T3 transformation: The reason of this process is that most of the image energy lies within $\mathrm{T}_{1}$ component and also human eye is more sensitive towards luminance change than color changes. So is good to work in $\mathrm{T}_{1} \mathrm{~T}_{2} \mathrm{~T}_{3}$ domain and to treat these three components separately. 
3. DWT procedure: In this step, the process applies the DWT on the original image up to three levels in order to obtain the vector of wavelet coefficients. We note that we used mother wave bior4.4 detailed in 10 .

4. Thresholding: simply, if the absolute values of NonZero Wavelet Coefficients (NZWC) are less than a given THreshold (TH), these coefficients are eliminated.

In this step we employed the bisection algorithm similar to that in 5] to control the PNSR in advance with a precision of convergence $\epsilon$ (it is chosen equal to 0.01).

5. Quantification: In this step, the NZWC are quantified by a linear quantification of size $Q$ bits. The objective of this quantification is to reduce the number of bits necessary to the representation of these coefficients. The quantification of the NZWC is done according to the formula [5]:

$$
\mathrm{QNZWC}=\left\lfloor 1+\frac{\mathrm{NZWC}-\mathrm{NZWC}_{\mathrm{Min}}}{\mathrm{NZWC}_{\mathrm{Max}}-\mathrm{NZWC}_{\mathrm{Min}}} \times\left(2^{Q}-2\right)\right\rfloor .
$$

where: $\left\lfloor\right.$ represents the nearer value, $\mathrm{NZWC}_{\mathrm{Min}}$ is the minimal value of

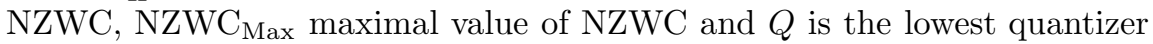
resolution.

In this step, every NZWC is quantized to become a Quantified NZWC (QNZWC) with the lowest possible resolution.

6. The Two Role Encoder (TRE): In this step, the process encodes the quantified coefficients in the zig-zag sequence by lossless encoding TRE [5]. The QNZWC is coded by a non negative integer of width equal $(Q+1)$ bits .

The thresholding step yields to many long run of zeros, each one is replaced by only one TRE code of $(Q+1)$ bits. The minimum run of zeros that is allowed to be coded by a TRE code is 1 , the maximum value is $2^{Q}-1$.

7. Arithmetic encoder: The concatenation of all vectors produces a global vector that is compressed by means of the arithmetic encoder.

\subsection{GA-DWT Based Decompression Phase}

Decompression is just the inverse process of compression as indicated in Fig. 3.

\section{Experimental Results and Performance Comparison}

In order to assess and test the robustness and the efficiency of the proposed GADWT based approach, we have used the well-known color images : Airplane, Peppers, Lena of size $512 \times 512$ for each one and Girl, Couple and House of size $256 \times 256$ for each one.

The results reported in Table 2; show the efficiency in performance of our GA-DWT based approach in the $\mathrm{T}_{1} \mathrm{~T}_{2} \mathrm{~T}_{3}$ color space.

The curves given on the Fig.4 illustrate that the bpp and the PSNR obtained in the $\mathrm{T}_{1} \mathrm{~T}_{2} \mathrm{~T}_{3}$ space are better than of the direct application on the RGB space. Therefore these results confirm that the $T_{1} T_{2} T_{3}$ color space is more suitable for compression. 
Table 2. Performances in the RGB and $T_{1} T_{2} T_{3}$ space for the different quantizer width

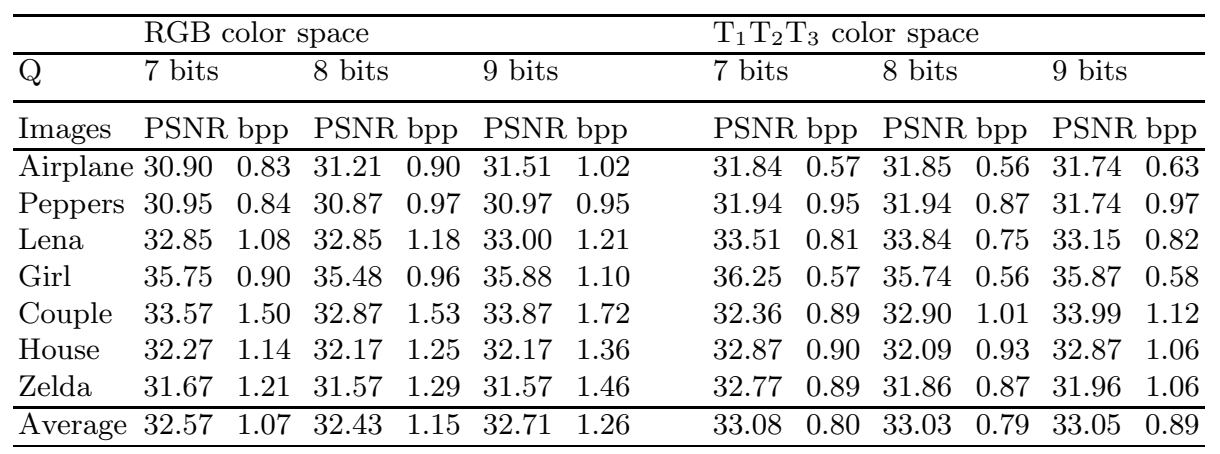



(a)

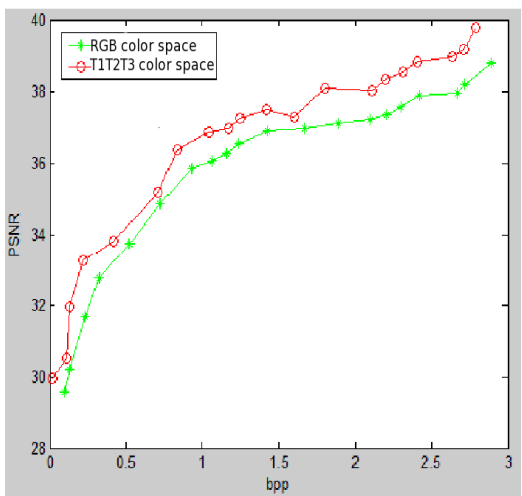

(b)

Fig. 4. Performances in the RGB and $T_{1} T_{2} T_{3}$ space applied for Lena and Girl color images: (a) Lena, (b) Girl

Figure 5 gives visual and quantitative results of the proposed method.

Comparative results of the recent published algorithms in [3] 4] and our proposed algorithm are presented in Table 3.

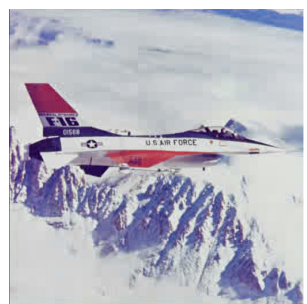

(a)

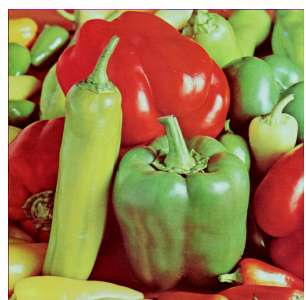

(b)

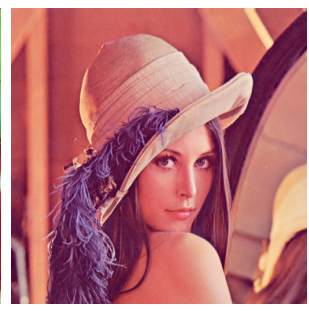

(c)

Fig. 5. Reconstructed images: (a) Airplane ( $\mathrm{PSNR}=31.16, \mathrm{bpp}=0.49)$, (b) Peppers $(\mathrm{PSNR}=31.20, \mathrm{bpp}=0.83),(\mathrm{c})$ Lena $(\mathrm{PSNR}=32.76, \mathrm{bpp}=0.66)$ 
Table 3, shown clearly that the results of our approach are particularly powerful compared to the CBTC-PF and CDABS.

Table 3. Performances comparison between the proposed method, CDABS and the CBTC-PF algorithm

\begin{tabular}{llllllll}
\hline \multirow{2}{*}{ Images } & \multicolumn{2}{c}{ Proposed method } & \multicolumn{2}{c}{ CDABS [4] } & \multicolumn{2}{c}{ CBTC-PF } \\
\cline { 2 - 8 } & \multicolumn{3}{l}{ PNSR bpp } & 30.38 & 0.59 & 30.36 & 1.04 \\
\hline Airplane & 31.16 & 0.49 & 30.05 & 0.80 & 30.15 & 1.50 \\
Peppers & 31.20 & 0.83 & 31.97 & 0.81 & 31.93 & 1.17 \\
Lena & 32.76 & 0.66 & 35.00 & 0.45 & 35.13 & 0.60 \\
Cirl & 35.90 & 0.41 & 32.28 & 0.92 & 32.44 & 1.00 \\
Couple & 32.87 & 0.89 & 31.72 & 0.82 & 31.79 & 1.20 \\
House & 32.10 & 0.83 & 31.33 & 0.87 & 31.31 & 1.12 \\
Zelda & 31.98 & 0.76 & 31.82 & 0.75 & 31.87 & 1.09 \\
\hline Average & 32.57 & 0.69 & & & &
\end{tabular}

\section{Conclusion}

In this paper, we have proposed a new color image compression method based on DWT and an appropriate GA. This approach is based on the fact that there are an infinite number of possible color spaces instead of the RGB channels. The best of these other color spaces are deriving by using a GA based on the DWT transform. Indeed, we apply our proposed GA-DWT approach in order to find a more suitable space referred to as $T_{1} T_{2} T_{3}$ for each image from the given RGB image. Thus, this new GA-DWT approach has the ability to build this base $\mathrm{T}_{1} \mathrm{~T}_{2} \mathrm{~T}_{3}$, which $\mathrm{T}_{1}$ energy is more maximized than that of $\mathrm{T}_{2}$ and $\mathrm{T}_{3}$, which permits a more effective compression because the information is concentrated in the plan $\mathrm{T}_{1}$. Thus, our GA-DWT have the ability to compresses more effectively $\mathrm{T}_{2}$ and $\mathrm{T}_{3}$ in order to eliminate the intercolor planes correlation.

The evaluation tests and experimental results obtained on different color images, showed clearly that the $\mathrm{T}_{1} \mathrm{~T}_{2} \mathrm{~T}_{3}$ color space is better than RGB in general. In addition, the obtained results are rather satisfactory compared to the CBTC$\mathrm{PF}$ and CDABS.

Acknowledgments. The authors thank Mr. Redha Benzid for his original ideas and encouragement which helped in obtaining the results.

\section{References}

1. Khalid, S.: Introduction to data compression. Elsevier, San Francisco (2006)

2. Zixiang, X., Kannan, R., Orchard, M.T., Ya-Qin, Z.: A Comparative study of DCTand Wavelet-based image coding. IEEE Transactions on Circuits and Systems for Video Thechnology 9, 692-695 (1999)

3. Chandra Dhara, B., Chanda, B.: Color image compression based on block truncation coding using pattern fitting principle. Pattern Recognition 40, 2408-2417 (2007) 
4. Douak, F., Benzid, R., Benoudjit, N.: Color image compression algorithm based on the DCT transform combined to an adaptive block scanning. AEU - International Journal of Electronics and Communications 65, 16-26 (2011)

5. Benzid, R., Marir, F., Bouguechal, N.-E.: Electrocardiogram compression method based on the adaptive wavelet coefficients quantization combined to a modified Two-Role Encoder. IEEE Signal Processing Letters 14, 373-376 (2007)

6. Varun, S., Vinod, K.: Coding of DWT coefficients using Run-length coding and Huffman coding for the purpose of color image compression. World Academy of Science, Engineering and Technology 62, 696-699 (2012)

7. Bhardwaj, A., Ali, R.: Image compression using modified fast haar wavelet transform. World Applied Sciences Journal 7, 647-653 (2009)

8. Elharar, E., Stren, A., Hadar, O., Jvidi, B.: A Hybrid compression method for integral images using discrete wavelet transform and discrete cosine transform. Journal of Display Technology 5, 1-5 (2007)

9. Piotr, P., Agnieszka, L.: The haar wavelet transform in digital image processing: Its status and achievements. Machine Graphics and Vision 13, 79-98 (2004)

10. RajKumar, T.M.P., Mrityunjaya Latte, V.: Performance evolution of various wavelet families in spiht image compression technique. European Journal of Scientific Research 59, 14-21 (2011)

11. Ghamisi, P., Santha devi, P., Phil, M.: Efficient wavelet based image compression technique for wireless communication. International Journal of Innovative Technology and Creative Engineering 1, 53-59 (2011)

12. Alkholidi, A., Cottour, A., Alfalou, A., Hamam, H., Keryer, G.: Real-time optical 2D wavelet transform based on the JPEG 2000 standards. European Physical Journal Applied Physics (EPJ AP) 44, 261-272 (2008)

13. Holland, J.H.: Adaptation in natural and artificial systems. University of Michigan Press, Ann Arbor (1975)

14. Goldberg David, E.: Genetic Algorithms in Search, Optimization and Machine Learning. Addison-Wesley Longman Publishing Co., Inc., Boston (1989)

15. Chakrapani, Y., Soundara Rajan, K.: Genetic algorithm applied to fractal image compression. ARPN Journal of Engineering and Applied Sciences 4, 53-58 (2009)

16. Lifeng, X., Liangbin, Z.: A study of fractal image compression based on an improved genetic algorithm. International Journal of Nonlinear Science 3, 116-124 (2007)

17. Alkholidi, A., Alfalou, A., Hamam, H.: A new approach for optical colored image compression using the JPEG standards. Signal Processing 87, 569-583 (2007)

18. Chan, K.Y., Stich, D., Voth, A.G.: Real-time image compression for high-speed particle tracking. Review of Scientific Instruments 78 (2007)

19. Andrew, C., Peter, F., Hartmut, P., Carlos, F.: Genetic Algorithm TOOLBOX For Use with MATLAB, Version 1.2 Users Guide, http://www.shef.ac.uk/acse/research/ecrg/gat.html 